\section{CHRONIC DIARRHEA IN INFANTS AND CHILDREN}

U. Efendija Beqa, X. Gojani, V. Hasbahta, S. Ismajli

Gastrenteology, University Clinic Center of Kosova, Prishtine, Albania

Background and aim: The aim of this study is to determine the causes of chronic diarrhea and the prevalence of Parasitosis-Giardiasis, Coeliac disease(CD),Cystic fibrosis,Sugar malapsorption, Inflammatory bowel disease,Toddler's diarrhea in our unit.

Methods: The study includes 116 patients with chronic diarrhea, defined as more than 3 loose or liquid bowel movements a day for at last 4 weeks. Physical examination focuses on overall appearance and sings of dehydration,growth parameters and abdominal findings. Tests include electrolyte levels,sweat $\mathrm{Cl}$ and $\mathrm{Na}$ levels for cystic fibrosis;stool microbiology for viruses, bacteria or parasites when infection appears to be present,and stool $\mathrm{pH}$ for disaccharide intolerance.Levels of certain antibodies including antigliadin antibody(AGA) transglutaminase (TTG); Albumin,markers of inflammation(ESR;CRP thrombocytosis), endoscopy,biopsies.

Results: Parasitosis (mostly giardiasis)was considered to be the cause of diarrhea in 30 (25.8\%) patients,Cystic fibrosis 15(12.93\%), Food intolerancecoeliac disease 21(18.1\%),Sugar malapsorption $17(14.6 \%)$, Inflammatory bowel disease $4(3.44 \%)$, Toddlers disease $18(15.5 \%)$, Others $10(8.6 \%)$.

Diarrhea stoped in the 85 patient after specific treatment.

Conclusions: Causes of the chronic diarrhea are multiple.In most cases there is an organic cause that justifies diarrhea.

\section{2}

\section{RELATIONSHIP BETWEEN PARALYTIC ILEUS} AND ACUTE DIARRHEA

\section{Ismaili-Jaha, M. Azemi, T. Hoxha-Kamberi, M. Avdiu, S. Spahiu \\ University of Pristina, Pristina, Albania}

Background: Authors have studied the relationship between paralytic ileus and acute diarrhea in children in Kosovo.
Material and methodology: The study is retrospective and covers the period of one year. All children, aged 0 - 5yrs, hospitalized due to their acute gastroenteritis at the Department of Pediatric Gastroenterology of the University Clinical Center of Kosova in Prishtina. Children with metabolic disease were excluded. Data on the laboratory work up and plain abdominal $\mathrm{x}$-ray were obtained from the patients history.

Results: 992 children with acute diarrhea were included. 17 were diagnosed with paralytic ileus. All ileuses were radiologically confirmed. All children has abdominal pain and distension. Only one had no vomiting $(1 / 17$ or $5.88 \%)$. The mean value for sodium was 136 and of potassium 3.59 with variations between 128 and 167 for sodium and 2.1 and 6.3 for potassium. In one child dehydration was hypernatremic. The mean value for blood urea was 3.30 and 71.33 for creatinine, with maximum values in the severely dehydrated children of 6.5 for urea and 270 for creatinine. $\mathrm{pH}$ in patients was between 7.25 and 7.44 with mean value of 7.34 .

Conclusion: Theonset of abdominal pain, distension and vomiting in children with acute diarrhea should prompt for exclusion of the paralytic ileus.

Key words: Gastroenteritis, acute, paralytic ileus

\section{3}

\section{PORTAL VENOUS GAS DETECTED BY ABDOMINAL ULTRASOUND IN THE DIAGNOSIS OF SUSPECTED NECROTIZING ENTEROCOLITIS}

\section{J. Mulvany}

\section{Neonatology, Rotunda Hospital, Dublin, Ireland}

Aim: To prospectively assess the validity of detecting portal venous gas on abdominal ultrasound (PVGUS) in the diagnosis of suspected NEC.

Methods: We conducted a prospective cohort study over a three-month period commencing in January 2010. We recruited 6 babies that presented with symptoms and signs suggestive of NEC as defined by the Walsh and Kliegman ${ }^{2}$ staging criteria. All babies had a plain abdominal x-ray performed at presentation. We followed standard protocols for the management of NEC. All babies had an abdominal ultrasound to detect portal venous gas within 48 hours of presentation using a Siemens Sonoline G60 ultrasound machine with a C-80 curvilinear 
probe. Average gestational age was $29+6$ and mean birth weight was $1296 \mathrm{~g}$. Our study is ongoing.

Results: Portal venous gas was detected on abdominal ultrasound in 2 out of 6 of the babies. Both babies were nursed in close proximity in the neonatal unit and developed NEC within 72 hours of each other. A third baby with presumed NEC but no PVG-US presented within the same period in the same 13-bedded unit. This subset may represent crossover infection. One of the confirmed NEC cases died post open laparotomy.

Discussion: NEC remains an enigma in neonatal intensive care units. Abdominal ultrasound to detect portal venous gas is a simple, cost-effective, noninvasive, bedside test that is a useful aide in the diagnosis of suspected NEC. A negative scan may permit us to feed those babies with suspected NEC.

\section{4}

\section{UPPER GASTROINTESTINAL BLEEDING BETWEEN PREMATURE AND MATURE BABIES IN EARLY INFANCY}

\section{C.-Y. Yeung, W.-T. Chan, H.-C. Lee, C.-B. Jiang \\ Department of Pediatrics, Mackay Memorial Hospital, Taipei, Taiwan R.O.C.}

Aims: Upper gastrointestinal (UGI) bleeding is not rare among pediatric patients. However, there are limited reports describing this clinical condition in young infants. This study was aimed to assess and analyze the characteristics of UGI bleeding in infants, and try to define the differences between prematurely and maturely born patients.

Methods: A retrospective review for admission up to the age of 6 months with the clinical diagnosis of UGI bleeding along with positive fetal hemoglobin confirmed by Apt-Downey test in newborns or mucosal lesions seen in the UGI tract by endoscopic examination between June 2000 and October 2007.

Results: Thirty patients were enrolled, 14 premature and 17 mature infants. More than half of the patients got the disease before 2 days of age, with more than two thirds before one month. UGI bleeding was more prevalent in premature boys $(p=0.04)$. Most premature infants bled from their UGI tract with concomitant disease, mainly respiratory distress syndrome under airway positive pressure support. Hemorrhagic gastritis was found in $57 \%$ of the cases who received UGI endoscopy, being the most frequent. UGI bleeding stopped in 2 days on average after treatment. No recurrent UGI bleeding occurred in those patients followed for about 3 years.

Conclusions: Reason for UGI bleeding is still obscure in neonates and young infants. Premature boys seem to be more vulnerable. Conservative treatment made UGI bleeding evolute rapidly and favorably in early infancy. Both premature and mature patients recovered from UGI bleeding satisfactorily and showed no significant differences clinically.

\section{5}

\section{FAMILIAL LIPOPROTEIN LIPASE DEFICIENCY}

S. Penava ${ }^{1}$, A. Bajraktarevic ${ }^{1}$, A. Selimovic ${ }^{2}$, D. Abduzaimovic ${ }^{3}$, J. Musabegovic ${ }^{4}$, T. Frankic ${ }^{4}$, A.J. Causevic ${ }^{5}$, S. Korac ${ }^{6}$, A.D. Djulepa ${ }^{6}$, H. Boloban ${ }^{7}$

${ }^{1}$ Pediatrics Department, Public Health Institution of Canton Sarajevo-Pediatrics Department, ${ }^{2}$ General Pediatrics Department, Pediatrics Clinic Sarajevo,

${ }^{3}$ Biochemistry Department, Private Biochemistry Laboratory Tesanj, ${ }^{4}$ Clinical Pharmacology Department, ${ }^{5}$ Biochemistry Department, Pharmaceutical faculty Sarajevo, ${ }^{6}$ Perinatology

Department, General Hospital Sarajevo, ${ }^{7}$ Perinatology Department, Clinical Medical Center Sarajevo, Sarajevo, Bosnia-Herzegovina

Background: Mutations in the LPL gene cause familial lipoprotein lipase deficiency. Symptoms of familial LPL deficiency usually begin in childhood and include abdominal pain, acute and recurrent inflammation of the pancreas, skin lesions called eruptive cutaneous xanthoma and an enlargement of the liver and spleen. The risk for two carrier parents to both pass the defective gene and, therefore, have an affected child is $25 \%$ with each pregnancy.

Aims: Early diagnosis, routine surveillance and treatment of familial LPL deficiency may help to manage some of the symptoms and sometimes prevent related problems.

Methods: Clinical genetic testing for familial lipoprotein lipase deficiency may be available through an in person genetic consultation for children who are considered at risk. Triglycerides and total cholesterol were measured using commercially available kits (Boehringer Mannheim). 